\title{
Density-orientation coupling for a microcontinuum approach to nematic liquid crystals subject to electric field
}

Received: 20 April 2020 / Accepted: 3 December 2020 / Published online: 17 January 2021 (C) The Author(s) 2021

\begin{abstract}
A microcontinuum description of compressible liquid crystals is examined accounting for a constitutive model based on mass microdensity. As a first point, we discuss the effectiveness of the micropolar theory on compressible continua, which is limited to static problems. Then, by a micromorphic representation of mass density, we show the consistence of some classical constitutive models for compressible nematic liquid crystals and remark their connection with the microinertia tensor. After the analysis of a constitutive micropolar model, we discuss a static problem for a layer of compressible nematic liquid crystal in a planar configuration. The effects of an applied electric potential are considered remarking the coupling of density distribution with the molecular orientation.
\end{abstract}

Keywords Nematic liquid crystals · Microcontinuum theory $\cdot$ Compressible continua $\cdot$ Electroelastic coupling

\section{Introduction}

The large field of investigations on liquid crystals is mainly based on classical continuum theories which account for molecular structures described by the "director" vector variable $[1,2]$. This theory has been developed exploiting suitable constitutive laws depending on gradients of the vector variable [3-6]. Nematic liquid crystals have been discussed in connection with various phenomena, including static and dynamic problems which involve electromagneto-elastic and acoustic effects. In particular, a relevant phenomenon in static problems concerns the Freedericksz transition for electric or magnetic fields that induce molecular orientation. Many investigations regard the behavior of incompressible liquid crystals, but relevant results on acoustic waves are obtained by a dynamical analysis of compressible liquid crystals where constitutive assumptions account for a dependence on density gradients [7-9].

An alternative continuum approach to liquid crystals is given by the micromorphic theory which links molecular orientation to microstructural deformation [10,11]. In particular, this approach is based on a micropolar theory that describes deformations due to microrotation. Electromagnetic effects are also considered in this theory through the proper extension of constitutive laws. A further investigation on these effects has been recently proposed on the basis of a consistent micromorphic description of polarization and magnetization [1214]. Unlike previous articles, in this work we are interested in a microcontinuum description of compressible liquid crystals, in order to examine the coupling of mass density with molecular orientation due to an applied electric field. The first part of the paper concerns an essential question about the compressibility condition.

Communicated by Andreas Öchsner.

M. Romeo ( $\varangle)$

DIMA, Università degli Studi di Genova, via Dodecaneso 35, 16146 Genova, Italy

E-mail: maurizio.romeo@unige.it 
We show that the classical micropolar theory can be applied to static problems of compressible materials but its effectiveness in dynamical problems is limited to the incompressible cases. A further result concerns the formulation of a constitutive law for compressible materials with a dependence on microdensity and its gradients. This model turns out to support and extend some constitutive laws proposed within the director theory of liquid crystals $[7,8,15]$. In particular, the connection of these laws with the microinertia tensor is remarked. Then we reduce our model to the micropolar static theory, in view of its application to the problem of a nematic homogeneous layer subject to an electric potential. The differential system which represents this problem is solved with an expansion technique based on a parameter which represents the electric potential intensity above the threshold of the Freedericksz transition. Our results are compatible with those obtained for analogous problems developed within the classical director theory $[15,16]$ where the density-orientation coupling is investigated.

\section{Microdensity and balance of mass}

The microcontinuum theory is based on the intrinsic structure of the continuum particle $\mathcal{P}$ occupying the volume $\Delta V$, which is described by the vector position $\xi$ with respect to the center of mass $\mathbf{x}$ of $\mathcal{P}$. Denoting by $\mathbf{X}$ and $\boldsymbol{\Xi}$, respectively, the vector positions of $\mathbf{x}$ and $\boldsymbol{\xi}$ in the reference configuration, the deformation and microdeformation tensors $\mathbf{F}=\nabla_{\mathbf{X}} \mathbf{x}(\mathbf{X}, t)$ and $\chi=\nabla_{\Xi} \xi(\mathbf{X}, \boldsymbol{\Xi}, t)$ account for the kinematic micromorphic description. In components

$$
F_{h K}=x_{h, K}, \quad \chi_{j H}=\xi_{j, H}
$$

where capital indices denote components in the reference configuration. These tensors are assumed to be smooth invertible functions. Then, material properties of elastic materials can be referred to the microdensity of mass $\hat{\rho}(\mathbf{x}, \boldsymbol{\xi}, t)$ such that the mass density $\rho$ is given by its mean value on $\Delta V$,

$$
\rho(\mathbf{x}, t)=\frac{1}{\Delta V} \int_{\Delta V} \hat{\rho}(\mathbf{x}+\xi, t) \mathrm{d} v .
$$

For any volume's portion of the macro-continuum, mass is conserved. This implies the following wellknown balance law

$$
\dot{\rho}+\rho \nabla \cdot \mathbf{v}=0,
$$

where superposed dot denotes the material time derivative. Here we look for a corresponding law on microdensity. We start from the conservation of the microelement's mass, consistent with the previous micromorphic description, by imposing

$$
\frac{\mathrm{d}}{\mathrm{d} t} \int_{\Delta V} \hat{\rho}(\mathbf{x}+\xi, t) \mathrm{d} v=0 .
$$
have

Accounting for the dependence of the microdisplacement function $\xi$, posing $J=\operatorname{det} \chi$ and $\mathfrak{X}=\chi^{-1}$, we

$$
\dot{J}=J \operatorname{tr}(\mathfrak{X} \dot{\chi}) .
$$

The application of the Reynold's transport theorem to the microstructure volume element yields

$$
\begin{aligned}
\frac{\mathrm{d}}{\mathrm{d} t} \int_{\Delta V} \hat{\rho}(\mathbf{x}+\boldsymbol{\xi}, t) \mathrm{d} v & =\frac{\mathrm{d}}{\mathrm{d} t} \int_{\Delta \mathcal{V}} \hat{\rho}[\mathbf{x}(\mathbf{X}, t)+\boldsymbol{\xi}(\mathbf{X}, \boldsymbol{\Xi}, t), t] J \mathrm{~d} v \\
& =\int_{\Delta \mathcal{V}}\left[\frac{\partial \hat{\rho}}{\partial t}+\nabla \hat{\rho} \cdot(\mathbf{v}+\dot{\boldsymbol{\xi}})+\hat{\rho} \operatorname{tr}(\mathfrak{X} \dot{\chi})\right] J \mathrm{~d} v \\
& =\int_{\Delta V}\left[\frac{\partial \hat{\rho}}{\partial t}+\nabla \hat{\rho} \cdot(\mathbf{v}+\dot{\boldsymbol{\xi}})+\hat{\rho} \operatorname{tr}(\mathfrak{X} \dot{\chi})\right] \mathrm{d} v,
\end{aligned}
$$

where $\Delta \mathcal{V}$ and $\mathrm{d} v$ denote quantities in the reference configuration. Then, Eq. (2.3) implies

$$
\int_{\Delta V}[\dot{\hat{\rho}}+\hat{\rho} \operatorname{tr}(\mathfrak{X} \dot{\chi})] \mathrm{d} v=0 .
$$


We observe that this result must hold also for any subvolume $\Delta \hat{V} \subseteq \Delta V$ containing the particle's center of mass, so that, in view of the continuity of the integrand,

$$
\dot{\hat{\rho}}=-\hat{\rho} \operatorname{tr}(\mathfrak{X} \dot{\chi}) \text {. }
$$

This result will be exploited in the successive analysis. Now we link micromass and mass conservation laws. Portion of the macro-continuum, derivative of Eq. (2.1) and exploiting (2.3), we obtain

$$
\dot{\rho}=-\frac{\rho}{\Delta V} \int_{\Delta V} \operatorname{tr}(\mathfrak{X} \dot{\chi}) \mathrm{d} v
$$

Substituting this result into Eq. (2.2), we obtain the following constraint for mass conservation

$$
\nabla \cdot \mathbf{v}=\frac{1}{\Delta V} \int_{\Delta V} \operatorname{tr}(\mathfrak{X} \dot{\chi}) \mathrm{d} v
$$

A stricter condition arises from the classical approach of microcontinuum theory which is based on the following linear dependence of $\hat{\xi}$ from $\boldsymbol{\Xi}$,

$$
\hat{\xi}(\mathbf{X}, \boldsymbol{\Xi}, t)=\chi(\mathbf{X}, t) \boldsymbol{\Xi}
$$

In this case, introducing the microrotation tensor $\mathbf{N}$ such that $\dot{\xi}=\mathbf{N} \xi$ (see [17]), we get $\mathbf{N}=\dot{\chi} \mathfrak{X}$ and $\operatorname{tr}(\mathfrak{X} \dot{\chi})=\operatorname{tr}(\mathbf{N})$. This last quantity is independent of the microdisplacement variable and Eq. (2.7) reduces to

$$
\nabla \cdot \mathbf{v}=\operatorname{tr}(\mathbf{N})
$$

We observe that, owing to (2.4), the condition $\operatorname{tr}(\mathfrak{X} \dot{\chi})=0$ represents the micro-incompressibility restriction. Then, the previous results can be synthesized as follows.

Statement A sufficient condition for the dynamic incompressibility of a continuum, described by a micromorphic general theory where $\boldsymbol{\xi}=\hat{\boldsymbol{\xi}}(\mathbf{X}, \mathbf{\Xi}, t)$, is given by

$$
\operatorname{tr}(\mathfrak{X} \dot{\chi})=0 .
$$

Under the linear assumption $\xi=\chi(\mathbf{X}, t) \mathbf{\Xi}$, this is also a necessary condition (see also [17,18]).

Remark 1 The theory of micropolar continua is a restriction of the micromorphic approach, where the microdeformation tensor $\chi$, which describes a pure rotation, is an orthogonal tensor [17]. This implies $\mathfrak{X}=\chi^{T}$ so that the microrotation tensor is skew-symmetric,

$$
\mathbf{N}^{T}=-\mathbf{N}
$$

In this case $\operatorname{tr}(\mathbf{N})=0$ and Eq. (2.8) implies that the micropolar approach to problems of solid or fluid dynamics holds only for incompressible materials.

In the following, after the general constitutive description, we shall consider a static problem for compressible nematic liquid crystals exploiting the micropolar theory.

\section{Constitutive model of a compressible micromorphic material}

The starting point of the present constitutive model is the assumption that the mass density is involved in the elastic response of a compressible material through its microstructure. 
3.1 Mass density representation

The dependence of the free energy on the microdeformation tensor and its gradients is here extended to a dependence on the microdensity and its gradients on the basis of a simple expansion of $\rho$. In this respect, the first- and second-order moments of microdensity per unit mass are

$$
\frac{1}{\rho \Delta V} \int_{\Delta V} \hat{\rho}(\mathbf{x}+\xi, t) \xi \mathrm{d} v=0, \quad \mathcal{I}=\frac{1}{\rho \Delta V} \int_{\Delta V} \hat{\rho}(\mathbf{x}+\xi, t) \xi \otimes \xi \mathrm{d} v
$$

where the first equality arises from the property of the center of mass $\mathbf{x}$ and $(3.1)_{2}$ represents the microinertia tensor per unit mass. Expanding $\hat{\rho}$ about $\mathbf{x}$, up to second order of $\boldsymbol{\xi}$, we get

$$
\hat{\rho}(\mathbf{x}+\xi, t)=\hat{\rho}(\mathbf{x}, t)+\nabla \hat{\rho}(\mathbf{x}, t) \cdot \xi+\frac{1}{2} \xi \cdot \nabla(\nabla \hat{\rho})(\mathbf{x}, t) \xi+o\left(\xi^{2}\right),
$$

and integrating, we obtain the following approximation of (2.1),

$$
\rho(\mathbf{x}, t)=\hat{\rho}(\mathbf{x}, t)+\nabla \hat{\rho}(\mathbf{x}, t) \frac{1}{\Delta V} \int_{\Delta V} \xi \mathrm{d} v+\frac{1}{2} \nabla(\nabla \hat{\rho})(\mathbf{x}, t): \frac{1}{\Delta V} \int_{\Delta V} \xi \otimes \xi \mathrm{d} v .
$$

Again, exploiting Eq. (3.1),

$$
\begin{aligned}
\frac{1}{\Delta V} \int_{\Delta V} \xi \mathrm{d} v & =-\frac{1}{\hat{\rho}(\mathbf{x}, t)} \mathcal{I}(\mathbf{x}, t) \nabla \hat{\rho}(\mathbf{x}, t)+o\left[M\left(\xi^{2}\right)\right], \\
\frac{1}{\Delta V} \int_{\Delta V} \xi \otimes \xi \mathrm{d} v & =\mathcal{I}(\mathbf{x}, t)+o\left[M\left(\xi^{2}\right)\right],
\end{aligned}
$$

where $M\left(\xi^{k}\right)$ denotes microdensity moment of order $k$. Substituting into (3.3), we obtain the following expansion

$$
\rho=\hat{\rho}-\frac{1}{\hat{\rho}} \nabla \hat{\rho} \cdot \mathcal{I} \nabla \hat{\rho}+\frac{1}{2} \operatorname{tr}[(\nabla \nabla \hat{\rho}) \mathcal{I}]+o\left(M\left(\xi^{2}\right)\right) .
$$

This result demonstrates the essential role of microdensity, evaluated at $\mathbf{x}(\xi=0)$, and its gradients coupled with microinertia to represent mass density effects.

\subsection{Constitutive equations and balance laws}

The next step is the decomposition of free energy $\psi$ into a purely elastic contribution $U$ and a contribution $\Sigma$ due to the microdensity-deformation coupling, as

$$
\hat{\rho} \psi=\hat{\rho} \Sigma(\hat{\rho}, \nabla \hat{\rho}, \nabla \nabla \hat{\rho}, \mathcal{I}, \vartheta)+U(\mathcal{I}, \Upsilon \mathbf{r}, \vartheta)
$$

where $\vartheta$ is the temperature and $\Upsilon \Upsilon$ is the wryness tensor. In terms of the microdeformation tensor, we have

$$
\Upsilon_{k l m}=\chi_{k H, M} \mathfrak{X}_{H l} F_{M m}^{-1}, \quad \mathcal{I}_{i j}=\chi_{i H} \mathcal{J}_{H K} \chi_{j K},
$$

where $\mathcal{J}$ is the microinertia in the reference configuration

$$
\mathcal{J}=\frac{1}{\rho(\mathbf{X}) \Delta \mathcal{V}} \int_{\Delta \mathcal{V}} \hat{\rho}(\mathbf{X}+\boldsymbol{\Xi}) \boldsymbol{\Xi} \otimes \mathbf{\Xi} \mathrm{d} \nu .
$$

According to the general micromorphic theory $[17,19]$, we introduce the following microstrain tensors

$$
a_{k l}=v_{l, k}-N_{l k}, \quad b_{l m k}=N_{l m, k}, \quad c_{k l}=\frac{1}{2}\left(N_{k l}+N_{l k}\right)
$$

where $\mathbf{v}$ is the velocity vector, and denoting by $e$ and $\eta$ the energy and entropy densities, such that $\psi=e-\vartheta \eta$, we write the balance of energy and the entropy inequality as follows 


$$
\begin{aligned}
& -\hat{\rho} \dot{e}+t_{k l} a_{k l}+m_{k l m} b_{l m k}+s_{k l} c_{k l}+q_{k, k}+\hat{\rho} h=0, \\
& \hat{\rho} \dot{\eta}-\nabla \cdot\left(\frac{\mathbf{q}}{\vartheta}\right)-\hat{\rho} \frac{h}{\vartheta} \geq 0,
\end{aligned}
$$

where $\mathbf{q}$ and $h$ are the heat flux vector and the energy source density. Here we restrict to not viscous effects and the constitutive laws for the stress tensor $\mathbf{T}$, the couple stress tensor $\mathbf{M}$ and the symmetric microstress tensor $\mathbf{S}$ (in components, respectively $t_{k l}, m_{k l m}, s_{k l}$ ) are deduced by the Clausius-Duhem inequality obtained from (3.7)

$$
-\hat{\rho}(\dot{\psi}+\dot{\vartheta} \eta)+\frac{1}{\vartheta} q_{k} \theta_{, k}+t_{k l} a_{k l}+m_{k l m} b_{l m k}+s_{k l} c_{k l} \geq 0
$$

By the standard procedure, we evaluate the time derivative of $\psi$ and after substitution into (3.8), neglecting contributions of higher gradients of $\mathbf{v}$ we have

$$
\begin{aligned}
& \hat{\rho}^{2}\left(\frac{\partial \Sigma}{\partial \hat{\rho}}-\frac{1}{\hat{\rho}^{2}} U\right) v_{k, k}+\hat{\rho} \frac{\partial \Sigma}{\partial \hat{\rho}_{, k}}\left(\hat{\rho}_{, h} v_{h, k}+\rho_{, k} v_{h, h}\right)+\hat{\rho} \frac{\partial \Sigma}{\partial \hat{\rho}_{, k q}}\left(2 \hat{\rho}_{, h k} v_{h, q}+\hat{\rho}_{, k q} v_{h, h}\right) \\
& -\hat{\rho}\left(\frac{\partial \Sigma}{\partial \mathcal{I}_{i j}}+\frac{1}{\hat{\rho}} \frac{\partial U}{\partial \mathcal{I}_{i j}}\right)\left(N_{i h} \mathcal{I}_{h j}+N_{j h} \mathcal{I}_{h i}\right)-\frac{\partial U}{\partial \Upsilon_{k l m}}\left(N_{k l, m}+N_{k p} \Upsilon_{p l m}-\Upsilon_{k p m} N_{p l}-\Upsilon_{k l p} v_{p, m}\right) \\
& \quad-\hat{\rho}\left(\frac{\partial \Sigma}{\partial \vartheta}+\frac{1}{\hat{\rho}} \frac{\partial U}{\partial \vartheta}\right) \dot{\vartheta}-\hat{\rho} \eta \dot{\vartheta}+\frac{1}{\vartheta} q_{, k} \vartheta_{, k}+t_{k l} a_{k l}+m_{k l m} b_{l m k}+s_{k l} N_{k l} \geq 0 .
\end{aligned}
$$

Then, exploiting the arbitrariness of the dynamic variables $\nabla \mathbf{v}, \mathbf{N}$ and $\nabla \mathbf{N}$, we obtain the following results

$$
\begin{aligned}
t_{k l} & =-p \delta_{k l}-\hat{\rho} \frac{\partial \Sigma}{\partial \hat{\rho}_{, k}} \hat{\rho}_{, l}-2 \hat{\rho} \frac{\partial \Sigma}{\partial \hat{\rho}_{, r k}} \hat{\rho}_{, l r}-\frac{\partial U}{\partial \Upsilon_{r p k}} \Upsilon_{r p l}+U \delta_{k l}, \\
s_{k l} & =t_{k l}+2 \hat{\rho}\left(\frac{\partial \Sigma}{\partial \mathcal{I}_{k j}}+\frac{1}{\hat{\rho}} \frac{\partial U}{\partial \mathcal{I}_{k j}}\right) \mathcal{I}_{l j}-\frac{\partial U}{\partial \Upsilon_{h l m}} \Upsilon_{h k m}+\frac{\partial U}{\partial \Upsilon_{k j m}} \Upsilon_{l j m}, \\
m_{k l m} & =\frac{\partial U}{\partial \Upsilon_{l m k}}, \\
\eta & =-\frac{\partial \Sigma}{\partial \vartheta}-\frac{1}{\hat{\rho}} \frac{\partial U}{\partial \vartheta}, \quad \frac{1}{\vartheta} \mathbf{q} \cdot \nabla \vartheta \geq 0 .
\end{aligned}
$$

where

$$
p=\hat{\rho}^{2} \frac{\partial \Sigma}{\partial \hat{\rho}}+\hat{\rho} \frac{\partial \Sigma}{\partial \hat{\rho}_{, i}} \hat{\rho}_{, i}+\hat{\rho} \frac{\partial \Sigma}{\partial \hat{\rho}_{, i j}} \hat{\rho}_{, i j},
$$

is the contribution of pressure to stress.

Now, on the basis of the result (3.4), we introduce the following expression of $\Sigma$,

$$
\Sigma=\sigma(\hat{\rho})-\alpha_{1} \operatorname{tr}[(\nabla \nabla \hat{\rho}) \mathcal{I}]+\alpha_{2} \frac{1}{\hat{\rho}} \nabla \hat{\rho} \cdot \mathcal{I} \nabla \hat{\rho},
$$

where $\sigma$ is a positive smooth function of $\hat{\rho}$ and $\alpha_{1}, \alpha_{2}$ are positive real constant parameters. In the following, we shall consider isothermal problems excluding a dependence on temperature.

Here we focus on the contributions of $\Sigma$ to the constitutive laws, transferring the explicit form of the micro-elastic contribution $U$ to the discussion of the micropolar case in the next section. Then, Eqs. (3.9) and (3.10) (3.12) yield

$$
\begin{aligned}
t_{k l} & =-p \delta_{k l}+2 \alpha_{1} \hat{\rho} \mathcal{I}_{k h} \hat{\rho}_{, h l}-2 \alpha_{2} \mathcal{I}_{k h} \hat{\rho}_{, h} \hat{\rho}_{, l}+U \delta_{k l}-\frac{\partial U}{\partial \Upsilon_{r p k}} \Upsilon_{r p l} \\
s_{k l} & =2 \frac{\partial U}{\partial \mathcal{I}_{k j}} \mathcal{I}_{l j}-\frac{\partial U}{\partial \Upsilon_{h l m}} \Upsilon_{h k m}+\frac{\partial U}{\partial \Upsilon_{k h m}} \Upsilon_{l h m}-\frac{\partial U}{\partial \Upsilon_{h m k}} \Upsilon_{h m l}+(U-p) \delta_{k l} \\
p & =\hat{\rho}^{2} \frac{\partial \sigma}{\partial \hat{\rho}}-\alpha_{1} \hat{\rho} \mathcal{I} \nabla \nabla \hat{\rho}+\alpha_{2} \nabla \hat{\rho} \cdot \mathcal{I} \nabla \hat{\rho} .
\end{aligned}
$$

These results show the relevant role of microinertia in the contributions of the density gradients to the stress tensor. Since $\mathcal{I}$ depends on the microdeformation, the micromorphic model accounts for a non-rigid 
orientation of molecular particles, which is a specific interest when modeling compressible nematic liquid crystals. We observe that the required symmetry of $\mathbf{S}$ must be satisfied by the dependence of $U$ from the invariants of tensors $\mathcal{I}$ and $\Upsilon$ as remarked in [11] for the micropolar case of nematic liquid crystals which will be discussed in the next sections.

The balance of momentum and the balance of moment of momentum, for a micromorphic material, are respectively (see $[17,20])$

$$
\begin{aligned}
& \rho \dot{\mathbf{v}}=\rho \mathbf{f}+\mathbf{f}^{\mathrm{em}}+\nabla \cdot \mathbf{T}, \\
& \rho \boldsymbol{\sigma}=\rho(\nabla \mathbf{f}) \mathcal{I}+\mathbf{C}^{\mathrm{em}}+\mathbf{T}^{T}-\mathbf{S}+\nabla \cdot \mathbf{m},
\end{aligned}
$$

where the rate of spin inertia tensor $\sigma$ is given by

$$
\sigma=\dot{\mathbf{N}} \mathcal{I}+\mathbf{N N} \mathcal{I} .
$$

and $\mathbf{f}$ is a body force density. The additional force vector $\mathbf{f}^{\mathrm{em}}$ and couple tensor $\mathbf{C}^{\mathrm{em}}$, due to electromagnetic contributions, have been discussed in $[12,21]$ and its reduction to the micropolar case in the presence of an applied electric field are considered in the next section.

\subsection{Comparison with director theory}

In comparison with the current continuum theories of these materials, based on the classical "director" model, we can confirm some effective constitutive models for compressible nematic liquid crystals where, differently from the present approach, molecular inertia turns out to be independent on elastic deformation. We observe that, physically, $\hat{\rho}(\mathbf{x})$ is a mass density measure (its value at a particle's central point) so that Eq. (3.13) is comparable with a macro-continuum constitutive law for the mass density contribution to energy. In this respect, we observe that, denoting by $\mathbf{n}$ the unit vector of director theory for liquid crystals, the microinertia per unit mass of the purely rigid rotating molecular elements can be written as

$$
\mathcal{I}=I_{1} \mathbf{I}+I_{2} \mathbf{n} \otimes \mathbf{n}
$$

with $I_{1}=I_{\perp}, I_{2}=I_{\|}-I_{\perp}$, where $I_{\perp}$ and $I_{\|}$are the inertia moments with respect to an axis, respectively, orthogonal and parallel to $\mathbf{n}$. Substituting into (3.13) we have

$$
\Sigma=\sigma(\hat{\rho})-\alpha_{1}\left[I_{1} \Delta \hat{\rho}+I_{2} \mathbf{n} \cdot(\nabla \nabla \hat{\rho}) \mathbf{n}\right]+\alpha_{2} \frac{1}{\hat{\rho}}\left[I_{1}\|\nabla \hat{\rho}\|^{2}+I_{2}(\nabla \hat{\rho} \cdot \mathbf{n})^{2}\right] .
$$

Supposing $\alpha_{1}=\alpha_{2}=\alpha$, we obtain the following generalization of the energy density for the director-density coupling corresponding to the current models based on the microdensity,

$$
\Sigma=\sigma(\hat{\rho})+\alpha\left\{\frac{1}{\hat{\rho}}\left[I_{1}\|\nabla \hat{\rho}\|^{2}+I_{2}(\nabla \hat{\rho} \cdot \mathbf{n})^{2}\right]-I_{1} \Delta \hat{\rho}-I_{2} \mathbf{n} \cdot(\nabla \nabla \hat{\rho}) \mathbf{n}\right\} .
$$

Specifically, if we omit second-order gradients of density, Eq. (3.19) corresponds to the model proposed by Virga [7] where a nematic liquid crystal behaves as an anisotropic Korteweg fluid. The parameters $u_{1}$ and $u_{2}$ in [7], corresponding to $\alpha I_{1}$ and $\alpha I_{2}$, are required to obey the inequalities $u_{1} \geq 0$ and $u_{1}+u_{2} \geq 0$, which are automatically here satisfied since $I_{\perp}$ and $I_{\|}$are positive quantities. The additional second-order gradient contribution $\mathbf{n} \cdot(\nabla \nabla \rho) \mathbf{n}$ was previously introduced by Selinger et al. [8] and successively extended by Vitoriano [15] including the term $\|\nabla \rho\|^{2}$ to avoid a singularity on a boundary value problem. We observe that Eq. (3.19) also includes the term $\Delta \hat{\rho}$ which is expected to complete the second gradient inclusion. As an additional comment on this comparison, we remark that parameters $u_{1}$ and $u_{2}$, which play the role of acoustic susceptibilities in dynamic problems, are here identified with the inertial properties of molecular particles. Based on the results about the acoustic torque in the director-density coupling (see [7,22]), it is expected that the alignment of $\mathbf{n}$ along the wave number direction occurs for $u_{2}<0$. This is in coherence with the case in which the inertial moment $I_{\perp}$ be larger than $I_{\|}$. 


\section{Micropolar reduction for nematic liquid crystals}

Now we reduce the previous model to the micropolar theory, which will be applied to a static problem on nematic liquid crystals, coherently with the remark at the end of Sect. 2. In this case, from the definition of the wryness tensor (3.6) and the orthogonality of tensor $\chi$, we can introduce a new second-order wryness tensor $\gamma$

$$
\gamma_{k l}=-\frac{1}{2} \epsilon_{i j k} \Upsilon_{i j l}
$$

Due to the skew-symmetric property (2.9) for the the microrotation tensor $\mathbf{N}$ in the micropolar case, we have $c_{k l}=0$ and the contribution of the symmetric tensor $\mathbf{S}$ in (3.7), (3.8) vanishes. Then, the constitutive laws (3.14) and (3.11) reduce to

$$
\mathbf{T}=\hat{\mathbf{T}}+U \mathbf{I}-\boldsymbol{\mu} \boldsymbol{\gamma}, \quad m_{k l m}=-\frac{1}{2} \epsilon_{l m h} \mu_{k h}
$$

where

$$
\hat{\mathbf{T}}=-p \mathbf{I}+2 \alpha_{1} \hat{\rho} \mathcal{I}(\nabla \nabla \hat{\rho})-2 \alpha_{2}(\mathcal{I} \nabla \hat{\rho}) \otimes \nabla \hat{\rho},
$$

is the density contribution to the stress. The reduced second-order couple stress tensor $\mu$ is given by

$$
\mu_{i j}=\frac{\partial U}{\partial \gamma_{j i}} .
$$

According to the constitutive restrictions for the elastic contribution $U$, given in [11], we assume the following quadratic dependence on $\boldsymbol{\gamma}$,

$$
U=\frac{1}{2} B_{i j k l} \gamma_{i j} \gamma_{k l},
$$

where $\mathbf{B}$ depends on the microinertia tensor $\mathcal{I}$, as follows:

$$
\begin{aligned}
B_{h k j i}= & b_{1} \delta_{h k} \delta_{j i}+b_{2} \delta_{h i} \delta_{k j}+b_{3} \delta_{h j} \delta_{k i} \\
& +b_{4}\left(\mathcal{I}_{j i} \delta_{h k}+\mathcal{I}_{h k} \delta_{j i}\right)+b_{5}\left(\mathcal{I}_{h i} \delta_{k j}+\mathcal{I}_{k j} \delta_{h i}\right) \\
& +b_{6}\left(\mathcal{I}_{k i} \delta_{h j}+\mathcal{I}_{h j} \delta_{k i}\right)+b_{7}\left(\mathcal{I}_{k i} \delta_{h j}-\mathcal{I}_{h j} \delta_{k i}\right) .
\end{aligned}
$$

In the following, we shall consider a static problem, accounting for the effects of an applied electric potential. Then, the balance laws (3.17) and (3.18), under the static micropolar assumptions, give

$$
\begin{aligned}
& \nabla \cdot \mathbf{T}+\mathbf{f}^{\mathrm{em}}=\mathbf{0} \\
& \quad-\nabla \cdot \boldsymbol{\mu}+\mathbf{t}+\mathbf{c}^{\mathrm{em}}=\mathbf{0}
\end{aligned}
$$

where

$$
t_{i}=\epsilon_{i j k} t_{k j}, \quad c_{i}^{\mathrm{em}}=\epsilon_{i j k} C_{j k}^{\mathrm{em}} .
$$

4.1 Electric field contributions to the balance laws

A consistent microcontinuum approach to electroelastic interactions requires that, beside the material description based on mass microdensity, polarization effects due to electric charge distribution, must be included (see $[12,21])$. By introducing the charge microdensity $\hat{\zeta}(\mathbf{x}+\xi, t)$, we can write multipole densities. In particular, the zero-, first-, and second-order terms are

$$
\begin{aligned}
& \varsigma(\mathbf{x}, t)=\frac{1}{\Delta V} \int_{\Delta V} \hat{\zeta}(\mathbf{x}+\boldsymbol{\xi}, t) \mathrm{d} v, \quad \mathbf{p}(\mathbf{x}, t)=\frac{1}{\Delta V} \int_{\Delta V} \hat{\zeta}(\mathbf{x}+\xi, t) \xi \mathrm{d} v \\
& \mathbf{Q}(\mathbf{x}, t)=\frac{1}{\Delta V} \int_{\Delta V} \hat{\zeta}(\mathbf{x}+\xi, t) \xi \otimes \xi \mathrm{d} v
\end{aligned}
$$


which represent, respectively, electric charge, dipole and quadrupole densities. In the following, we will omit the dependence on $t$ for our static assumption. Introducing the elecromagnetic force microdensity $\hat{\mathbf{f}}^{\mathrm{em}}(\mathbf{x}+\boldsymbol{\xi})$, we write

$$
\mathbf{f}^{\mathrm{em}}(\mathbf{x})=\frac{1}{\Delta V} \int_{\Delta V} \hat{\mathbf{f}}^{\mathrm{em}}(\mathbf{x}+\xi) \mathrm{d} v, \quad \mathbf{C}^{\mathrm{em}}(\mathbf{x})=\frac{1}{\Delta V} \int_{\Delta V} \boldsymbol{\xi} \otimes \hat{\mathbf{f}}^{\mathrm{em}}(\mathbf{x}+\xi) \mathrm{d} v
$$

General deductions of these quantities are given in [20,21]. Here we consider only the contribution of an electric field $\mathbf{E}$ for a non conducting material where $\hat{\zeta}$ represents only bound charge microdensity with a mean value $\varsigma=0$ and we pose

$$
\hat{\mathbf{f}}^{\mathrm{em}}(\mathbf{x}+\xi)=\hat{\zeta}(\mathbf{x}+\xi) \mathbf{E}(\mathbf{x}+\xi) .
$$

By simply expanding this last quantity about $\boldsymbol{\xi}=\mathbf{0}$, using a procedure similar to that adopted in Sect. 3.1 and accounting for (4.5) with $\varsigma=0$, up to the second order of $\xi$, we obtain

$$
\begin{aligned}
\mathbf{f}^{\mathrm{em}} & =\mathbf{p} \cdot \nabla \mathbf{E}+\frac{1}{2}(\mathbf{Q} \nabla) \nabla \mathbf{E}, \\
\mathbf{C}^{\mathrm{em}} & =\mathbf{p} \otimes \mathbf{E}+\mathbf{Q}(\nabla \mathbf{E}) .
\end{aligned}
$$

The presence of the electric field implies an additional balance law corresponding to the Gauss' law for a zero charge density 5 ,

$$
\nabla \cdot \mathbf{D}=0,
$$

where $\mathbf{D}=\mathbf{E}+\mathbf{P}$ is the electric displacement and $\mathbf{P}$ represents the polarization. Here we use HeavisideLorentz units of electromagnetism. The micromorphic theory of electromagnetic materials allows to express polarization on the basis of electric multipoles (see [21,23]). Up to the second-order multipole, we have

$$
\mathbf{P}=\mathbf{p}-\frac{1}{2} \nabla \cdot \mathbf{Q}
$$

Due to the static assumption, we introduce the electric potential $\varphi$ so that $\mathbf{E}=-\nabla \varphi$. Accounting for the previous results, from Eqs. (4.4) to (4.7) we obtain the following set of governing equations for the present micropolar electroelastic model,

$$
\begin{aligned}
& \nabla \cdot \hat{\mathbf{T}}+\nabla U-\nabla \cdot(\boldsymbol{\mu} \boldsymbol{\gamma})-\mathbf{p} \cdot(\nabla \nabla \varphi)-\frac{1}{2}(\mathbf{Q} \nabla) \nabla \nabla \varphi=\mathbf{0}, \\
& -\nabla \cdot \boldsymbol{\mu}+\mathbf{t}-\mathbf{p} \times \nabla \varphi-(\mathbf{Q} \nabla) \times \nabla \varphi=\mathbf{0}, \\
& -\Delta \varphi+\nabla \cdot \mathbf{p}-\frac{1}{2} \nabla \cdot(\nabla \cdot \mathbf{Q})=0
\end{aligned}
$$

\subsection{Explicit microrotation formulation}

Here we describe the microrotation in terms of an angle $\theta$ and an axis of rotation $\mathbf{n}(\|\mathbf{n}\|=1)$ as proposed in the classical micropolar theory [17] so that the microdeformation tensor is given by

$$
\chi_{i H}=\cos \theta \delta_{i H}+\sin \theta \epsilon_{i j k} n_{j} \delta_{k H}+(1-\cos \theta) n_{i} n_{k} \delta_{k H} .
$$

Accordingly, the quantities $\gamma, \mathcal{I}, \mathbf{p}, \mathbf{Q}$, take the following explicit form

$$
\begin{aligned}
\gamma_{i j}= & n_{i} \theta, j+\sin \theta n_{i, j}+(1-\cos \theta) \epsilon_{i h k} n_{h} n_{k, j}, \\
\mathcal{I}_{i j}= & \cos ^{2} \theta \mathcal{J}_{i j}+\sin \theta \cos \theta\left(\epsilon_{i l k} \mathcal{J}_{k j}+\epsilon_{j l k} \mathcal{J}_{i k}\right) n_{l}+\cos \theta(1-\cos \theta)\left(n_{j} \mathcal{J}_{i k}+n_{i} \mathcal{J}_{k j}\right) n_{k} \\
& +\epsilon_{i h k} \epsilon_{j l m} \sin ^{2} \theta \mathcal{J}_{k m} n_{h} n_{l}+\sin \theta(1-\cos \theta)\left(\epsilon_{i l k} n_{j}+\epsilon_{j l k} n_{i}\right) \mathcal{J}_{k h} n_{h} n_{l} \\
& +(1-\cos \theta)^{2} \mathcal{J}_{l k} n_{l} n_{k} n_{i} n_{j}, \\
p_{i}= & \cos \theta \pi_{i}+\sin \theta \epsilon_{i j k} n_{j} \pi_{k}+(1-\cos \theta) n_{i} n_{k} \pi_{k},
\end{aligned}
$$




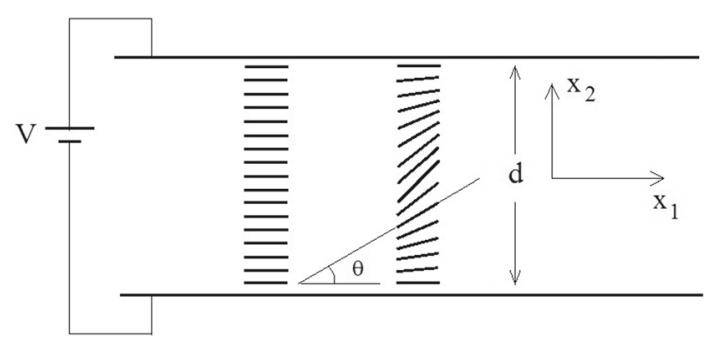

Fig. 1 Geometry of the nematic layer with homogeneous structure

$$
\begin{aligned}
Q_{i j}= & \cos ^{2} \theta \mathcal{Q}_{i j}+\sin \theta \cos \theta\left(\epsilon_{i l k} \mathcal{Q}_{k j}+\epsilon_{j l k} \mathcal{Q}_{i k}\right) n_{l}+\cos \theta(1-\cos \theta)\left(n_{j} \mathcal{Q}_{i k}+n_{i} \mathcal{Q}_{k j}\right) n_{k} \\
& +\epsilon_{i h k} \epsilon_{j l m} \sin ^{2} \theta \mathcal{Q}_{k m} n_{h} n_{l}+\sin \theta(1-\cos \theta)\left(\epsilon_{i l k} n_{j}+\epsilon_{j l k} n_{i}\right) \mathcal{Q}_{k h} n_{h} n_{l} \\
& +(1-\cos \theta)^{2} \mathcal{Q}_{l k} n_{l} n_{k} n_{i} n_{j}
\end{aligned}
$$

with $\pi_{i}=\mathbb{P}_{H} \delta_{i H}, \mathcal{J}_{i j}=\mathcal{J}_{H K} \delta_{i H} \delta_{j K}, \mathcal{Q}_{i j}=\mathbb{Q}_{H K} \delta_{i H} \delta_{j K}$, where $\mathbb{P}$ and $\mathbb{Q}$ are dipole and quadrupole densities in the reference configuration and $\delta_{i H}$ is the "shifter" between bases in the undeformed spatial and reference configurations. The quantities $\mathcal{J}, \pi, \mathcal{Q}$ represent material parameters of microcontinuum which characterize the anisotropic response to mechanical and electric loads. In particular, $\boldsymbol{\pi}$ describes a possible intrinsic electric dipole of the material particle.

\section{Electroelastic problem for a homogeneous nematic layer}

We apply our micropolar model to a static problem of a nematic liquid crystal layer of thickness $d$. We consider a homogeneous (planar) configuration with strong anchoring along two parallel rigid plane boundaries $S_{1}, S_{2}$. We refer this geometry to an orthonormal basis $\left\{\mathbf{e}_{1}, \mathbf{e}_{2}, \mathbf{e}_{3}\right\}$, where $\mathbf{e}_{2}$ is orthogonal to the plane boundaries so that $S_{1}$ and $S_{2}$ are described, respectively, by $x_{2}=-d / 2, x_{2}=d / 2$ and the axis $\mathbf{e}_{1}$ is parallel to the nematic orientation at the boundaries (see Fig. 1). An electric field is generated by the applied potentials $\varphi=-V / 2$, $\varphi=V / 2$, respectively, at $S_{1}$ and $S_{2}$. Our system is then reduced to a one-dimensional problem where the axis of microrotation is the fixed unit vector $\mathbf{n}=\mathbf{e}_{3}$ and the angle of particle's orientation $\theta$ with respect to $\mathbf{e}_{1}$, the electric potential $\varphi$ and the microdensity $\hat{\rho}$ depend on $x_{2}$.

We shall consider the micromorphic structure, referred to the undeformed homogeneous configuration, characterized by the following elements

$$
\mathcal{I}=\operatorname{diag}\left(\mathcal{J}_{\|}, \mathcal{J}_{\perp}, \mathcal{J}_{\perp}\right), \quad \mathcal{Q}=\operatorname{diag}\left(\mathcal{Q}_{\|}, \mathcal{Q}_{\perp}, \mathcal{Q}_{\perp}\right), \quad \pi_{i}=\hat{\pi} \delta_{i 2},
$$

where $\hat{\pi}$ is the intrinsic dipole arising from the applied electric field, so that $\hat{\pi}=-\varepsilon V / d$ where $\varepsilon$ denotes a positive dielectric relative permittivity. According to Eqs. (4.14)-(4.16), the microinertia and quadrupole tensors and the dipole vector are represented by

$$
\begin{aligned}
\mathcal{I} & =\left(\begin{array}{ccc}
\mathcal{J}_{\|} \cos ^{2} \theta+\mathcal{J}_{\perp} \sin ^{2} \theta & \mathcal{J}_{a} \sin \theta \cos \theta & 0 \\
\mathcal{J}_{a} \sin \theta \cos \theta & \mathcal{J}_{\perp} \cos ^{2} \theta+\mathcal{J}_{\|} \sin ^{2} \theta & 0 \\
0 & 0 & \mathcal{J}_{\perp}
\end{array}\right) \\
\mathcal{Q} & =\left(\begin{array}{ccc}
\mathcal{Q}_{\|} \cos ^{2} \theta+\mathcal{Q}_{\perp} \sin ^{2} \theta & \mathcal{Q}_{a} \sin \theta \cos \theta & 0 \\
\mathcal{Q}_{a} \sin \theta \cos \theta & \mathcal{Q}_{\perp} \cos ^{2} \theta+\mathcal{Q}_{\|} \sin ^{2} \theta & 0 \\
0 & 0 & \mathcal{Q}_{\perp}
\end{array}\right) \\
p_{1} & =\varepsilon \frac{V}{d} \sin \theta, \quad p_{2}=-\varepsilon \frac{V}{d} \cos \theta, \quad p_{3}=0,
\end{aligned}
$$

where $\mathcal{J}_{a}=\mathcal{J}_{\|}-\mathcal{J}_{\perp}, \mathcal{Q}_{a}=\mathcal{Q}_{\|}-\mathcal{Q}_{\perp}$. We observe that in this case the matrix of the wryness tensor (4.13) reduces to a single not vanishing entry $\gamma_{32}=\theta_{, 2}$. In view of a comparison with a similar problem discussed on the basis of the director theory (see [16]) corresponding to a different sign of dielectric anisotropy, we shall consider a simple linear dependence of the pressure contribution $p_{0}=\hat{\rho}^{2} \frac{\partial \sigma}{\partial \hat{\rho}}$ in (3.16),

$$
p_{0}(\hat{\rho})=\kappa \hat{\rho} .
$$


where $\kappa$ is a real positive parameter. In addition, according to the comparison of Sect. 3.3, we assume $\alpha_{1}=$ $\alpha_{2} \equiv \alpha$ in Eq. (3.13).

Under the present conditions, the micropolar model described in the previous section yields the following explicit form of balance Eqs. (4.9)-(4.11),

$$
\begin{aligned}
& -\kappa \hat{\rho}-3 \alpha \mathcal{J}_{a} \sin 2 \theta \theta_{, 2}\left(\hat{\rho} \hat{\rho}_{, 2}\right)_{, 2}-3 \alpha\left(\mathcal{J}_{\perp} \cos ^{2} \theta+\mathcal{J}_{\|} \sin ^{2} \theta\right)\left(\hat{\rho} \hat{\rho}_{, 2}\right)_{, 22} \\
& -\left(v_{b}+v_{\perp} \cos ^{2} \theta+v_{\|} \sin ^{2} \theta\right) \theta_{, 2} \theta, 22-\frac{1}{2} v_{a} \sin 2 \theta \theta_{, 2}^{3}+\varepsilon \frac{V}{d} \cos \theta \varphi, 22 \\
& -\frac{1}{2}\left(\mathcal{Q}_{\perp} \cos ^{2} \theta+\mathcal{Q}_{\|} \sin ^{2} \theta\right) \varphi_{, 222}=0 \\
& -\left(v_{b}+v_{\perp} \cos ^{2} \theta+v_{\|} \sin ^{2} \theta\right) \theta_{, 22}+v_{a} \sin 2 \theta \theta_{, 2}^{2}-\alpha \mathcal{J}_{a} \sin 2 \theta\left(\hat{\rho} \hat{\rho}_{, 2}\right)_{, 2} \\
& -\varepsilon \frac{V}{d} \sin \theta \varphi_{, 2}-\frac{1}{2} \mathcal{Q}_{a} \sin 2 \theta \varphi_{, 22}=0 \\
& -\varphi, 22+\varepsilon \frac{V}{d} \sin \theta \theta_{, 2}-\frac{1}{2} \mathcal{Q}_{a}\left(2 \cos 2 \theta \theta_{, 2}^{2}+\sin 2 \theta \theta_{, 22}\right)=0
\end{aligned}
$$

where we have specified

$$
v_{\perp}=\left(b_{6}+b_{7}\right) \mathcal{J}_{\perp}, \quad v_{\|}=\left(b_{6}+b_{7}\right) \mathcal{J}_{\|}, \quad v_{b}=b_{3}+\left(b_{6}-b_{7}\right) \mathcal{J}_{\perp}, \quad v_{a}=v_{\|}-v_{\perp} .
$$

In addition, Eq. (3.4) gives the following expression for the mass density,

$$
\rho=\hat{\rho}-\frac{1}{\hat{\rho}}\left(\mathcal{J}_{\perp} \cos ^{2} \theta+\mathcal{J}_{\|} \sin ^{2} \theta\right)\left(\hat{\rho}_{, 2}^{2}-\frac{1}{2} \hat{\rho} \hat{\rho}, 22\right) .
$$

The strong anchoring constraint at $S_{1}$ and $S_{2}$ requires the following boundary conditions for the present problem,

$$
\theta(-d / 2)=\theta(d / 2)=0, \quad \hat{\rho}(-d / 2)=\hat{\rho}(d / 2)=\hat{\rho}_{0}, \quad \varphi(-d / 2)=-\varphi(d / 2)=-V / 2 .
$$

A further restriction arises from the requirement of mass conservation which, in this one-dimensional problem implies

$$
\int_{-d / 2}^{d / 2} \rho\left(x_{2}\right) \mathrm{d} x_{2}=c
$$

where $c$ is a positive constant.

\subsection{Dimensionless formulation and trivial solution}

As a first step of the present analysis, we introduce the following dimensionless variables

$$
y=\frac{x_{2}}{d}, \quad \phi=\frac{\varphi}{V}, \quad \varrho=\frac{\hat{\rho}}{\hat{\rho}_{0}}
$$

and define the following dimensionless parameters

$$
\overline{\mathcal{J}}_{k}=\frac{\mathcal{J}_{k}}{d^{2}}, \quad \overline{\mathcal{Q}}_{k}=\frac{\mathcal{Q}_{k}}{V}, \quad \bar{v}_{k}=\frac{v_{k}}{\alpha \hat{\rho}_{0}^{2} d^{2}}, \quad \overline{v_{b}}=\frac{v_{b}}{\alpha \hat{\rho}_{0}^{2} d^{2}}, \quad \bar{\kappa}=\frac{\kappa}{\alpha \hat{\rho}_{0}}, \quad \beta=\frac{V^{2}}{\alpha \hat{\rho}_{0}^{2} d^{2}}
$$

where index $k$ replaces all symbols $\|, \perp, a$. Denoting by a prime the derivative with respect to $y$, we obtain the following dimensionless equations from (5.2)-(5.4),

$$
\begin{aligned}
& -\bar{\kappa} \varrho^{\prime}-\overline{\mathcal{J}}_{a} \sin 2 \theta \theta^{\prime}\left(\varrho \varrho^{\prime \prime}+\varrho^{\prime 2}\right)-\left(\overline{\mathcal{J}}_{\perp} \cos ^{2} \theta+\overline{\mathcal{J}}_{\|} \sin ^{2} \theta\right)\left(\varrho \varrho^{\prime \prime \prime}+3 \varrho^{\prime} \varrho^{\prime \prime}\right) \\
& -\left(\bar{v}_{b}+\bar{v}_{\perp} \cos ^{2} \theta+\bar{v}_{\|} \sin ^{2} \theta\right) \theta^{\prime} \theta^{\prime \prime}-\frac{1}{2} \bar{v}_{a} \sin 2 \theta \theta^{\prime 3}+\varepsilon \beta \cos \theta \phi^{\prime \prime} \\
& -\frac{1}{2} \beta\left(\overline{\mathcal{Q}}_{\perp} \cos ^{2} \theta+\overline{\mathcal{Q}}_{\|} \sin ^{2} \theta\right) \phi^{\prime \prime \prime}=0
\end{aligned}
$$




$$
\begin{aligned}
& -\left(\bar{v}_{b}+\bar{v}_{\perp} \cos ^{2} \theta+\bar{v}_{\|} \sin ^{2} \theta\right) \theta^{\prime \prime}+\bar{v}_{a} \sin 2 \theta \theta^{\prime 2} \\
& -\overline{\mathcal{J}}_{a} \sin 2 \theta\left(\varrho \varrho^{\prime \prime}+\varrho^{\prime 2}\right)-\varepsilon \beta \sin \theta \phi^{\prime}-\frac{1}{2} \overline{\mathcal{Q}}_{a} \sin 2 \theta \phi^{\prime \prime}=0 \\
& -\phi^{\prime \prime}+\varepsilon \sin \theta \theta^{\prime}-\frac{1}{2} \overline{\mathcal{Q}}_{a}\left(2 \cos 2 \theta \theta^{\prime 2}+\sin 2 \theta \theta^{\prime \prime}\right)=0,
\end{aligned}
$$

with boundary conditions

$$
\theta(-1 / 2)=\theta(1 / 2)=0, \quad \varrho(-1 / 2)=\varrho(1 / 2)=1, \quad \phi(-1 / 2)=-\phi(1 / 2)=-\frac{1}{2} .
$$

In addition, posing $\bar{\rho}=\rho / \hat{\rho}_{0}$, Eq. (5.5) takes the following dimensionless form

$$
\bar{\rho}=\varrho-\frac{1}{\varrho}\left(\overline{\mathcal{J}}_{\perp} \cos ^{2} \theta+\overline{\mathcal{J}}_{\|} \sin ^{2} \theta\right)\left(\varrho^{\prime 2}-\frac{1}{2} \varrho \varrho^{\prime \prime}\right) .
$$

A trivial solution of Eqs. (5.8)-(5.11), satisfying the boundary conditions, is given by $\theta(y)=0, \quad \varrho(y)=$ $1, \quad \phi(y)=y$, corresponding to

$$
\theta\left(x_{2}\right)=0, \quad \rho\left(x_{2}\right)=\hat{\rho}\left(x_{2}\right)=\hat{\rho}_{0}, \quad \varphi\left(x_{2}\right)=V x_{2} .
$$

The existence of a not vanishing solution for $\theta$ arises from the Freedericksz transition as discussed in the next subsection. We finally observe that the existence of the trivial solution (5.12) implies that for all possible solutions, the mass conservation constraint (5.7) requires $c=\hat{\rho}_{0} d$ and reduces to

$$
\int_{-1 / 2}^{1 / 2} \bar{\rho}(y) \mathrm{d} y=1 .
$$

\subsection{Linearization approach and threshold potential}

In order to obtain not trivial solutions, we proceed with a linear analysis by introducing a threshold value $V_{c}$ of the applied electric potential, such that a bifurcation from the trivial solution occurs for $V \geq V_{c}$. Since we are interested in a comparison of the micropolar approach with some results obtained by the director theory, as proposed in the reference [16], we expand the dependent variables of our problem in terms of the parameter $\eta=\sqrt{V / V_{c}-1}$

$$
\theta(y)=\sum_{h=1}^{\infty} \eta^{h} \theta_{h}(y), \quad \varrho(y)=1+\sum_{h=1}^{\infty} \eta^{h} \varrho_{h}(y), \quad \phi(y)=y+\sum_{h=1}^{\infty} \eta^{h} \phi_{h}(y),
$$

accounting for the boundary conditions, which imply vanishing values of $\theta_{h}, \varrho_{h}, \phi_{h}$ at the boundaries $y= \pm \frac{1}{2}$. As a first step, we note that by a simple integration, Eq. (5.10) reduces to

$$
-\phi^{\prime}-\varepsilon \cos \theta-\frac{1}{2} \overline{\mathcal{Q}} \sin 2 \theta \theta^{\prime}=\mathcal{E},
$$

where $\mathcal{E}$ is a constant. In this subsection and the following one, we proceed to discuss Eqs. (5.8), (5.9) and (5.14) by considering the various orders of the previous expansions and on the basis of a corresponding expression of the integration constant, $\mathcal{E}=\mathcal{E}_{0}+\sum_{h=1}^{\infty} \mathcal{E}_{h}$, where $\mathcal{E}_{0}=-1-\varepsilon$. After substitution of (5.14) into (5.9), we obtain

$$
\begin{aligned}
& -\left(\bar{v}_{b}+\bar{v}_{\perp} \cos ^{2} \theta+\bar{v}_{\|} \sin ^{2} \theta\right) \theta^{\prime \prime}+\bar{v}_{a} \sin 2 \theta \theta^{\prime 2}-\overline{\mathcal{J}}_{a} \sin 2 \theta\left(\varrho \varrho^{\prime \prime}+\varrho^{\prime 2}\right) \\
& +\varepsilon \beta \sin \theta\left(\varepsilon \cos \theta+\frac{1}{2} \overline{\mathcal{Q}}_{a} \sin 2 \theta \theta^{\prime}+\mathcal{E}\right) \\
& -\frac{1}{2} \overline{\mathcal{Q}}_{a} \sin 2 \theta\left[\varepsilon \sin \theta \theta^{\prime}-\frac{1}{2} \overline{\mathcal{Q}}\left(2 \cos 2 \theta \theta^{\prime 2}+\sin 2 \theta \theta^{\prime \prime}\right)\right]=0 .
\end{aligned}
$$


Up to the first order in $\eta$, this last equation gives

$$
\left(\bar{v}_{b}+\bar{v}_{\perp}\right) \theta_{1}^{\prime \prime}+\varepsilon \beta \theta_{1}=0,
$$

which admits the not vanishing solution

$$
\theta_{1}(y)=A \cos (\pi y) \quad \text { if } \quad \frac{\varepsilon \beta}{\bar{v}_{b}+\bar{v}_{\perp}}=\pi^{2},
$$

where $A$ is a constant amplitude to be determined. The condition in (5.17) gives the following threshold potential

$$
V_{c}=\pi \sqrt{\frac{v_{b}+v_{\perp}}{\varepsilon}},
$$

which, accounting for the connection of $v_{b}+v_{\perp}$ with the Frank constant $K_{1}$ (see for example [11]) turns out to be the classical critical potential of the Freedericksz transition for a homogeneous nematic layer. In the next analysis, we shall account for the connection of the parameter $\beta$ with $\eta$

$$
\beta=\left(1+\eta^{2}\right)^{2} \beta_{0}, \quad \text { with } \quad \beta_{0}=\frac{V_{c}}{\alpha \hat{\rho}_{0}^{2} d^{2}} .
$$

5.3 Orientation-density coupling induced by the electric potential

At the first order of Eq. (5.8) and the second order of Eq. (5.9), we obtain the following system

$$
\begin{aligned}
& \bar{\kappa} \varrho_{1}^{\prime}+\overline{\mathcal{J}}_{\perp} \varrho_{1}^{\prime \prime \prime}=0, \\
& \left(\bar{v}_{b}+\bar{v}_{\perp}\right) \theta_{2}^{\prime \prime}+2 \overline{\mathcal{J}}_{a} \theta_{1} \varrho_{1}^{\prime \prime}+\varepsilon \beta_{0} \theta_{2}=0
\end{aligned}
$$

which, exploiting Eq. (5.17) and the pertinent boundary conditions, gives the following simple solution

$$
\varrho_{1}(y)=0, \quad \theta_{2}(y)=B \cos (\pi y),
$$

where $B$ is an unknown constant. With respect to Eq. (5.14), at the first order, we obtain $\phi_{1}(y)=0$ and at the second order, in view of the previous result on $\theta_{1}$, we get $\mathcal{E}_{2}=\varepsilon A^{2} / 4$ and

$$
\phi_{2}(y)=A^{2}\left[\frac{\varepsilon}{8 \pi} \sin (2 \pi y)-\frac{\overline{\mathcal{Q}}_{a}}{2} \cos ^{2}(\pi y)\right]
$$

In order to obtain the first non-vanishing higher-order term for $\varrho$, we must consider higher orders for Eqs. (5.8) and (5.9). In view of the previous result, at the second order of Eq. (5.8), we have

$$
\bar{\kappa} \varrho_{2}^{\prime}+\overline{\mathcal{J}}_{\perp} \varrho_{2}^{\prime \prime \prime}+A^{2}\left[H_{1} \sin (2 \pi y)-H_{2} \cos (2 \pi y)\right]=0,
$$

where

$$
H_{1}=\frac{\pi}{2}\left[\left(\bar{v}_{b}+\bar{v}_{\perp}-2 \beta_{0} \overline{\mathcal{Q}}_{\perp} \overline{\mathcal{Q}}_{a}\right) \pi^{2}+\varepsilon^{2} \beta_{0}\right], \quad H_{2}=\varepsilon \beta_{0} \pi^{2}\left(\overline{\mathcal{Q}}_{a}+\frac{1}{2} \overline{\mathcal{Q}}_{\perp}\right) .
$$

The solution of Eq. (5.19) is

$$
\varrho_{2}(y)=K_{1} \cos (\lambda y)+K+\frac{1}{\lambda^{2}-4 \pi^{2}} \frac{A^{2}}{\pi \overline{\mathcal{J}}_{\perp}}\left[H_{1} \cos (2 \pi y)+H_{2} \sin (2 \pi y)\right]
$$

where

$$
\lambda=\sqrt{\frac{\bar{\kappa}}{\overline{\mathcal{J}}_{\perp}}}, \quad K=\frac{H_{1}}{2 \pi \overline{\mathcal{J}}_{\perp}} \frac{A^{2}}{\lambda^{2}-4 \pi^{2}}-K_{1} \cos (\lambda / 2) .
$$


In order to evaluate the constant $K_{1}$, we exploit the condition (5.13), expressing $\bar{\rho}$ up to the second order from Eq. (5.11),

$$
\bar{\rho}=1+\eta^{2}\left(1+\frac{1}{2} \overline{\mathcal{J}}_{\perp} \varrho_{2}^{\prime \prime}\right)+o\left(\eta^{2}\right)
$$

and obtain

$$
K_{1}=\left[\lambda \overline{\mathcal{J}}_{\perp} \sin (\lambda / 2)\right]^{-1}
$$

To complete the results up to the lowest significant order in $\eta$, we must deduce the constant $A$. This is possible by considering Eq. (5.9) at the third order,

$$
\begin{aligned}
\theta_{3}^{\prime \prime}+\pi^{2} \theta_{3}= & \frac{A \cos (\pi y)}{\bar{v}_{b}+\bar{v}_{\perp}}\left\{\varepsilon \beta_{0}\left(1+\frac{1}{4} \varepsilon A^{2}\right)-2 \overline{\mathcal{J}}_{a} \varrho_{2}^{\prime \prime}\right. \\
& \left.+\pi^{2} A^{2}\left[\bar{v}_{\|}+\left(\bar{v}_{a}-\bar{v}_{\perp}\right) \sin ^{2}(\pi y)-\overline{\mathcal{Q}}_{a}^{2} \cos (2 \pi y)\right]-\frac{\pi}{2} A^{2} \varepsilon \overline{\mathcal{Q}}_{a}\left(\beta_{0}-1\right) \sin (2 \pi y)\right\}
\end{aligned}
$$

We do not derive the explicit form of the solution $\theta_{3}$ and we simply report the following result obtained by imposing the boundary conditions $\theta_{3}(-1 / 2)=\theta_{3}(1 / 2)=0$,

$$
A= \pm 2 \sqrt{6 \frac{4 \pi^{2}-\lambda^{2}}{\pi^{2}-\lambda^{2}} \frac{\lambda \pi^{2} \overline{\mathcal{J}}_{a} \cot (\lambda / 2)+\left(\pi^{2}-\lambda^{2}\right) \varepsilon^{2} \beta_{0} \overline{\mathcal{J}}_{\perp}}{\left(4 \pi^{2}-\lambda^{2}\right) M_{\perp}+8 \pi^{2} M_{a}}}
$$

where

$$
M_{\perp}=\overline{\mathcal{J}}_{\perp}\left[4 \pi^{2}\left(\overline{\mathcal{Q}}_{a}^{2}+2 \bar{v}_{\perp}-2 \bar{v}_{\|}\right)-3 \varepsilon^{2} \beta_{0}\right], \quad M_{a}=\overline{\mathcal{J}}_{a}\left[1+2 \pi^{2} \beta_{0} \overline{\mathcal{Q}}_{a} \overline{\mathcal{Q}}_{\perp}+\pi^{2}\left(\bar{v}_{b}+\bar{v}_{\perp}\right)\right] .
$$

We finally observe that the consistency of a third-order evaluation of Eqs. (5.10) and (5.14) gives $B=0$ and $\phi_{3}(y)=0$. We conclude that, up to the lowest order in $\eta$ for our non trivial solution, $\theta(y)=A \cos (\pi y)$ and from (5.18), (5.20) and (5.21), we achieve the following results,

$$
\begin{aligned}
\phi(y) & =y+\eta^{2} A^{2}\left[\frac{\varepsilon}{8 \pi} \sin (2 \pi y)-\frac{\overline{\mathcal{Q}}_{a}}{2} \cos ^{2}(\pi y)\right] \\
\bar{\rho} & =1+\eta^{2}\left\{1-\frac{1}{2} \bar{\kappa} K_{1} \cos (\lambda y)-\frac{2 \pi A^{2}}{\lambda^{2}-4 \pi^{2}}\left[H_{1} \cos (2 \pi y)+H_{2} \sin (2 \pi y)\right]\right\}
\end{aligned}
$$

Comments Accounting for a translation $z=y+\frac{1}{2}$, our results can be compared with some results about the director-density coupling in nematic liquid crystals $[15,16]$. Although we are considering an alternative configuration of polarization of these studies where the main induced dipole is oriented along the director, some analogies can be remarked. Firstly, we note that the smallest-order nonzero contributions of electric potential $\phi$ and mass density $\bar{\rho}$ are the same as those found in [16]. A second-order contribution of $\theta$ vanishes as found in [15] but not discussed in [16]. Secondly, if we neglect the effects of quadrupole contribution to polarization, the quantities $\overline{\mathcal{Q}}_{a}$ and $H_{2}$ vanish and the behavior of $\phi$ corresponds to that obtained in [16]. If in addition, as modeled in [15], we neglect the contribution of $p_{0}(\kappa=0)$, we obtain the same result for $\bar{\rho}$, which, in this case, reads

$$
\bar{\rho}=1+\eta^{2} \frac{12}{\pi} \frac{\varepsilon^{2} \beta_{0} \overline{\mathcal{J}}_{\perp}}{M_{\perp}+2 M_{a}} \cos (2 \pi y)
$$

In this respect, we note that a possible not trivial contribution of pressure effects appears in the term proportional to $\cos (\lambda y)$ in (5.25), which can be viewed as an extension of the results in [16]. We finally observe that the contribution of electric quadrupole in (5.24), (5.25) accounts for a more detailed dependence of polarization on molecular orientation as remarked in previous results [13]. In particular, it gives a quadratic dependence of the electric potential on $\theta$.

In Figs. 2 and 3, we report the results of the dimensionless electric potential and density variation obtained in (5.24), (5.25). The data of various parameters are chosen according to some mechanical micropolar molecular 


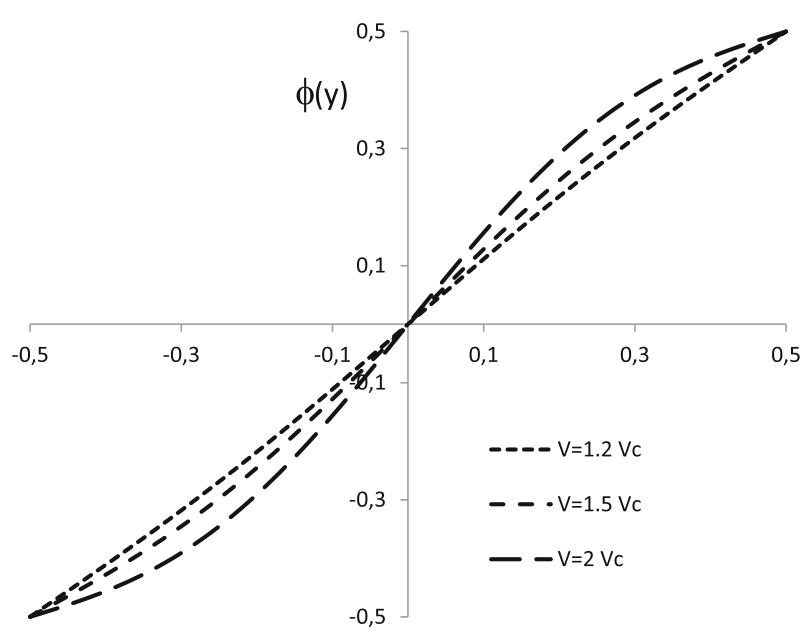

Fig. 2 Dimensionless electric potential $\phi$ versus dimensionless spatial coordinate $y$ for different values of the applied potential V

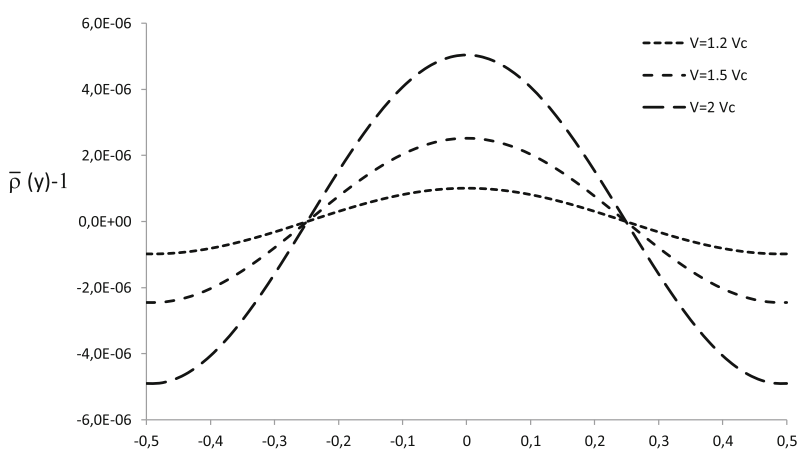

Fig. 3 Dimensionless variation of the mass density with respect to the undeformed configuration for different values of the applied potential $V$

models (see [17]) and electromagnetic descriptions of liquid crystals (see [24]). Specifically, the following data are chosen,

$$
\begin{aligned}
& \hat{\rho}_{0}=210^{3}, \quad \mathcal{J}_{\|}=\frac{1}{3} \mathcal{J}_{\perp}=10^{-19} \mathrm{~m}^{2}, \quad \mathcal{Q}_{\|}=-710^{-11} \mathrm{~cm}^{-1}, \quad \mathcal{Q}_{\perp}=-910^{-11} \mathrm{~cm}^{-1}, \\
& v_{\perp}=210^{-10} \mathrm{~N}, \quad v_{\|}=10^{-11} \mathrm{~N}, \quad v_{b}=10^{-9} \mathrm{~N}, \quad \varepsilon=0.1
\end{aligned}
$$

by considering a layer of thickness $d=10^{-4} \mathrm{~m}$ and choosing $\alpha=10^{2}$. As expected, a full spatially symmetric behavior of $\theta, \phi$ and $\bar{\rho}$ is obtained in the present approximation. Figure 2 also shows that a maximum intensity of the electric field occurs at $d=0$ where dipole intensity $p_{2}$ is minimized.

\section{Concluding remarks}

The first part of this work (Sect. 2), focused on the determination of the incompressibility condition, reveals the incompatibility of a micropolar mechanical theory for dynamical problems of compressible materials. In this regard we recall the partially effective results of this approach to the propagation of acoustic waves in nematic liquid crystals discussed in [25] where the result of a non dispersive wave mode is not consistent with experimental data and other nematoacoustic theories [7,26]. However, as shown in Sect. 3, the more general micromorphic theory, turns out to support some constitutive models of compressible liquid crystals, revealing a fundamental dependence of some material parameters on microinertia.

As remarked previously, the reduced micropolar model is here applied to a static problem of compressible liquid crystal, by considering a homogeneous configuration of a nematic layer subject to an electric field. 
In this case, the microrotation formulation allows a description of the coupling of mass density distribution and molecular orientation due to a dielectric response of the nematic structure. A simple comparison with a classical approach, based on the director model, shows that the more refined microcontinuum approach is compatible with the approximated results of the director theory on the differential problem of an electroelastic nematic layer. On the basis of the present result, further applications to dynamical problems on compressible materials are possible. In particular, the dispersion of acoustic waves is an interesting question that will be investigated.

Funding Open Access funding provided by Università degli Studi di Genova.

Open Access This article is licensed under a Creative Commons Attribution 4.0 International License, which permits use, sharing, adaptation, distribution and reproduction in any medium or format, as long as you give appropriate credit to the original author(s) and the source, provide a link to the Creative Commons licence, and indicate if changes were made. The images or other third party material in this article are included in the article's Creative Commons licence, unless indicated otherwise in a credit line to the material. If material is not included in the article's Creative Commons licence and your intended use is not permitted by statutory regulation or exceeds the permitted use, you will need to obtain permission directly from the copyright holder. To view a copy of this licence, visit http://creativecommons.org/licenses/by/4.0/.

\section{References}

1. Oseen, W.C.: The theory of liquid crystals. Trans. Faraday Soc. 29, 883-899 (1933)

2. Frank, F.C.: On the theory of liquid crystals. Discuss. Faraday Soc. 25, 19-28 (1958)

3. Ericksen, J.L.: Conservation laws for liquid crystals. Trans. Soc. Rheol. 5, 23-34 (1961)

4. Ericksen, J.L.: Equilibrium theory of liquid crystals. Adv. Liq. Cryst. 2, 233-298 (1976)

5. Leslie, F.M.: Some constitutive equations for liquid crystals. Arch. Rat. Mech. Anal. 28, 265-283 (1968)

6. Leslie, F.M.: Continuum theory for nematic liquid crystals. Contin. Mech. Thermodyn. 4, 167-175 (1992)

7. Virga, E.G.: Variational theory for nematoacoustics. Phys. Rev. E 80, 031705 (2009)

8. Selinger, J.V., Spector, M.S., Greanya, V.A., Weslowski, B.T., Shenoy, D.K., Shashidhar, R.: Acoustic realignment of nematic liquid crystals. Phys. Rev. E 66, 051708 (2002)

9. Vitoriano, C.: Generalization of the Ericksen-Leslie theory. Eur. Phys. J. E 40, 48 (2017)

10. Eringen, A.C.: A unified continuum theory of electrodynamics of liquid crystals. Int. J. Eng. Sci. 35, 1137-1157 (1997)

11. Eringen, A.C.: Microcontinuum Field Theories II-Fluent media. Springer-Verlag, New York (2001)

12. Romeo, M.: Micromorphic continuum model for electromagnetoelastic solids. Z. Angew. Math. Phys. 62, $513-527$ (2011)

13. Romeo, M.: Micropolar nematic model for polarized liquid crystals. Contin. Mech. Thermodyn. 30, 207-219 (2018)

14. Romeo, M.: Electromagnetic coupling in nematic liquid crystals modeled as microcontinua. Contin. Mech. Thermodyn. 31, 587-601 (2019)

15. Vitoriano, C.: Freedericksz transition in the director-density coupling theory. Phys. Rev. E 90, 032502 (2014)

16. De Matteis, G., Napoli, G.: Electric-field-induced density modulation in a nematic liquid crystal cell. SIAM J. Appl. Math. 73, 882-903 (2013)

17. Eringen, A.C.: Microcontinuum Field Theories I-Foundations and Solids. Springer-Verlag, New York (1999)

18. Romeo, M.: A microstretch description of electroelastic solids with application to plane waves. Math. Mech. Solids 24, 2181-2196 (2019)

19. Eringen, A.C., Suhubi, E.S.: Nonlinear theory of simple microelastic solids-I. Int. J. Eng. Sci. 2, 189-203 (1964)

20. Romeo, M.: A variational formulation for electroelasticity of microcontinua. Math. Mech. Solids. 20, 1234-1250 (2015)

21. Romeo, M.: A microstructure continuum approach to electromagneto-elastic conductors. Contin. Mech. Thermodyn. 28, 1807-1820 (2016)

22. De Matteis, G.: Acoustic torque acting upon nematic liquid crystals. Acta Appl. Math. 122, 205-223 (2012)

23. Romeo, M.: Polarization in dielectrics modeled as micromorphic continua. Z. Angew. Math. Phys. 66, $1233-1247$ (2015)

24. Villanueva-García, M., Robles, J., Martínez-Richa, A.: Quadrupolar moment calculations and mesomorphic character of model dimeric liquid crystals. Comput. Mater. Sci. 22, 300-308 (2001)

25. Lee, J.D., Eringen, A.C.: Wave propagation in nematic liquid crystals. J. Chem. Phys. 54, 5027-5034 (1971)

26. Wetsel, G.C., Speer, J.R.S., Lowry, B.A., Woodard, M.R.: Effects of magnetic field on attenuation of ultrasonic waves in a nematic liquid crystal. J. Appl. Phys. 43, 1495-1497 (1972)

Publisher's Note Springer Nature remains neutral with regard to jurisdictional claims in published maps and institutional affiliations. 